\title{
Гетероструктура квантово-каскадного детектора частотного диапазона 2.5 ТГц
}

(C) A.В. Бабичев ${ }^{1}$, E.C. Колодезный ${ }^{1}$, А.Г. Гладышев ${ }^{1}$, Д.В. Денисов ${ }^{2}$, A. Jollivet $^{3}$, P. Quach $^{3}$, Л.Я. Карачинский ${ }^{1,4,5}$, В.Н. Неведомский ${ }^{4}$, И.И. Новиков ${ }^{1,5}$, M. Tchernycheva ${ }^{3}$, F.H. Julien ${ }^{3}$, A.Ю. Eгоров ${ }^{5}$

${ }^{1}$ Национальный исследовательский университет ИТМО,

197101 Санкт-Петербург, Россия

${ }^{2}$ Санкт-Петербургский государственный электротехнический университет „ЛЭТИ“ им. В.И. Ульянова (Ленина), 197022 Санкт-Петербург, Россия

${ }^{3}$ Centre de Nanosciences et de Nanotechnologies (C2N), UMR 9001 CNRS, Universite Paris-Saclay, 91120 Palaiseau, France

${ }^{4}$ Физико-технический институт им. А.Ф. Иоффе Российской академии наук, 194021 Санкт-Петербург, Россия

${ }^{5} \mathrm{OOO} \mathrm{„Коннектор} \mathrm{Оптикс“,}$

194292 Санкт-Петербург, Россия

E-mail: a.babichev@mail.ioffe.ru

Поступила в Редакцию 15 октября 2021 г.

В окончательной редакции 29 октября 2021 г.

Принята к публикации 29 октября 2021 г.

\begin{abstract}
Предложена конструкция и реализована методом молекулярно-пучковой эпитаксии гетероструктура квантово-каскадного детектора частотного диапазона 2.5 ТГц. Для оптимизации толщин слоев периодов гетероструктуры использовался численный метод итерационного решения уравнения Шредингера-Пуассона в $k \cdot p$ формализме. Выращенная гетероструктура квантово-каскадного детектора показала высокое структурное совершенство, подтвержденное малыми значениями средней ширины на половине высоты пиков-сателлитов высоких порядков на рентгенодифракционных кривых качания, которые составили $(8.3 \pm 0.5)^{\prime \prime}$. Анализ темнопольных изображений, полученных методом просвечивающей электронной микроскопии, показал, что суммарная толщина слоев в периоде каскада составляет $137.3 \pm 6.9$ нм, что соответствует расчетной толщине слоев периода каскада гетероструктуры квантово-каскадного детектора.
\end{abstract}

Ключевые слова: сверхрешетки, квантово-каскадный детектор, эпитаксия, арсенид галлия.

DOI: $10.21883 /$ FTP.2022.03.52124.9750

\section{1. Введение}

Терагерцовая (ТГц) спектроскопия может обеспечить повышенную чувствительность в сравнении со спектроскопией ближней инфракрасной области спектра ввиду наличия колебательно-вращательных линий поглощения в терагерцовой области спектра ряда молекул. Сфера применения систем ТГц спектроскопии включает контроль технологических процессов на производствах [1], анализ состояния окружающей среды [2], a также оценку состояния здоровья человека $[3,4]$. В силу больших геометрических размеров и высокой стоимости коммерческие системы ТГц спектроскопии с временныб́м разрешением (THz TDS) [5] не получили широкого распространения, кроме того, их эффективность в частотном диапазоне выше 2 ТГц весьма ограничена.

Одним из перспективных подходов по созданию компактных систем ТГц спектроскопии считается развитие двойной гребенчатой спектроскопии (DCS) на основе квантово-каскадных лазеров (ККЛ) [1]. К настоящему моменту продемонстрированы ТГц ККЛ со значительной выходной оптической мощностью, работающие при температуре до $250 \mathrm{~K}$ [6], обладающие широким спектром усиления, что обеспечивает существенную пере- стройку длины волны излучения, что требуется для ТГЦ гребенчатой спектроскопии.

Несмотря на наличие быстродействующих детекторов ближнего [7] и среднего [8] инфракрасного (ИК) диапазонов, отсутствие быстродействующих детекторов дальнего ИК (ТГц диапазона), способных обеспечить интеграцию на чипе, существенно ограничивает развитие компактных систем двойной гребенчатой спектроскопии.

Полупроводниковые квантово-размерные ТГц детекторы на основе межподзонных переходов в силу малых времен жизни носителей заряда перспективны для реализации быстродействующих детекторов. ИК фотодетекторы на квантовых ямах (QWIP) в ТГц частотном диапазоне показывают высокую детектирующую способность, сопоставимую с чувствительностью болометров [9]. Однако существуют физические ограничения на работу ТГц QWIP при комнатной температуре. Более того, быстродействующие QWIP (частота отсечки на уровне 70 ГГц) были продемонстрированы на длинах волн $\sim 10$ мкм [8]. В то же время быстродействие ТГц QWIP не превышает 4.3ГГц [10,11]. Результаты по использованию гетероструктур ТГц ККЛ в качестве фотодетекторов представлены в работе [12], где достигнуто значение уровня мощности шума (NEP) на уровне 1.5 пВт/Гц ${ }^{0.5}$. 
Более перспективным видится развитие области фотовольтаических ТГц квантово-каскадных детекторов (ККД). В сравнении с QWIP структурами, работающими при смещении, ККД среднего инфракрасного (ИК) диапазона демонстрируют более низкие шумы (за счет отсутствия шума темнового тока, свойственного структурам QWIP) и возможность работы при повышенных температурах. В то же время в силу сложности численного моделирования зонной структуры ТГц ККД, транспорта носителей заряда, лишь одной научной группе удалось реализовать на практике ТГц ККД [13]. Авторами была продемонстрирована детектирующая способность $\sim 5 \cdot 10^{7} \mathrm{~cm} \cdot\left(\Gamma_{ц / \mathrm{BT}}\right)^{0.5}$ на частоте 3.5 ТГц и возможность работы при температуре $50 \mathrm{~K}$. В 2020 году представлены результаты по моделированию ТГц ККД с использованием байесовского алгоритма оптимизации [14], метода Монте-Карло в ансамбле совместно с применением генетического алгоритма оптимизации [15]. Данные результаты моделирования получили свое развитие в области ККД средней ИК области спектра [16].

В настоящей работе представлены первые результаты по моделированию и выращиванию методом молекулярно-пучковой эпитаксии гетероструктур квантовокаскадных детекторов частотного диапазона 2.5 ТГц.

\section{2. Экспериментальные образцы}

Конструкция периода каскада активной области ККД частотного диапазона 2.5 ТГц была рассчитана с использованием коммерчески доступного программного пакета NextNano ${ }^{\odot}$ [17] методом итерационного решения уравнения Шредингера-Пуассона в $k \cdot p$ формализме для температуры $4 \mathrm{~K}$ (см. рис. 1, a). Наряду с профилем зоны проводимости представлены квадраты волновых функций в слоях расчетной конструкции периода каскада. Расчет конструкции производился с целью реализации диагонального межподзонного поглощения в слое „активной“ квантовой ямы (КЯ) (обозначение на рис. $1, a$,active QW“). Энергия кванта между состояниями 1-5 составила 10.2 мэВ, между состояниями 1-611.7 мэВ, между состояниями $1-7-15.3$ мэВ.

После переходов с состояния 1 на состояния 5-7 электроны туннелируют в область экстрактора (область выброса носителей заряда). Проведена оптимизация параметров слоев с целью увеличения пространственного перекрытия огибающей волновых функций КЯ с областью выброса носителей заряда (состояниями 2-4), областью экстрактора (обозначение на рис. 1, $a$ „extractor" $)$. Легированными являются КЯ только в области экстрактора, что, с одной стороны, обеспечивает увеличение вероятности релаксации (выброса) электронов в область экстрактора (за счет электрон-примесного рассеяния), а с другой - одновременно уменьшает вероятность межподзонной релаксации носителей заряда в „активной“ КЯ. Как следствие, это приводит к увеличению эффективности переноса носителей заряда между

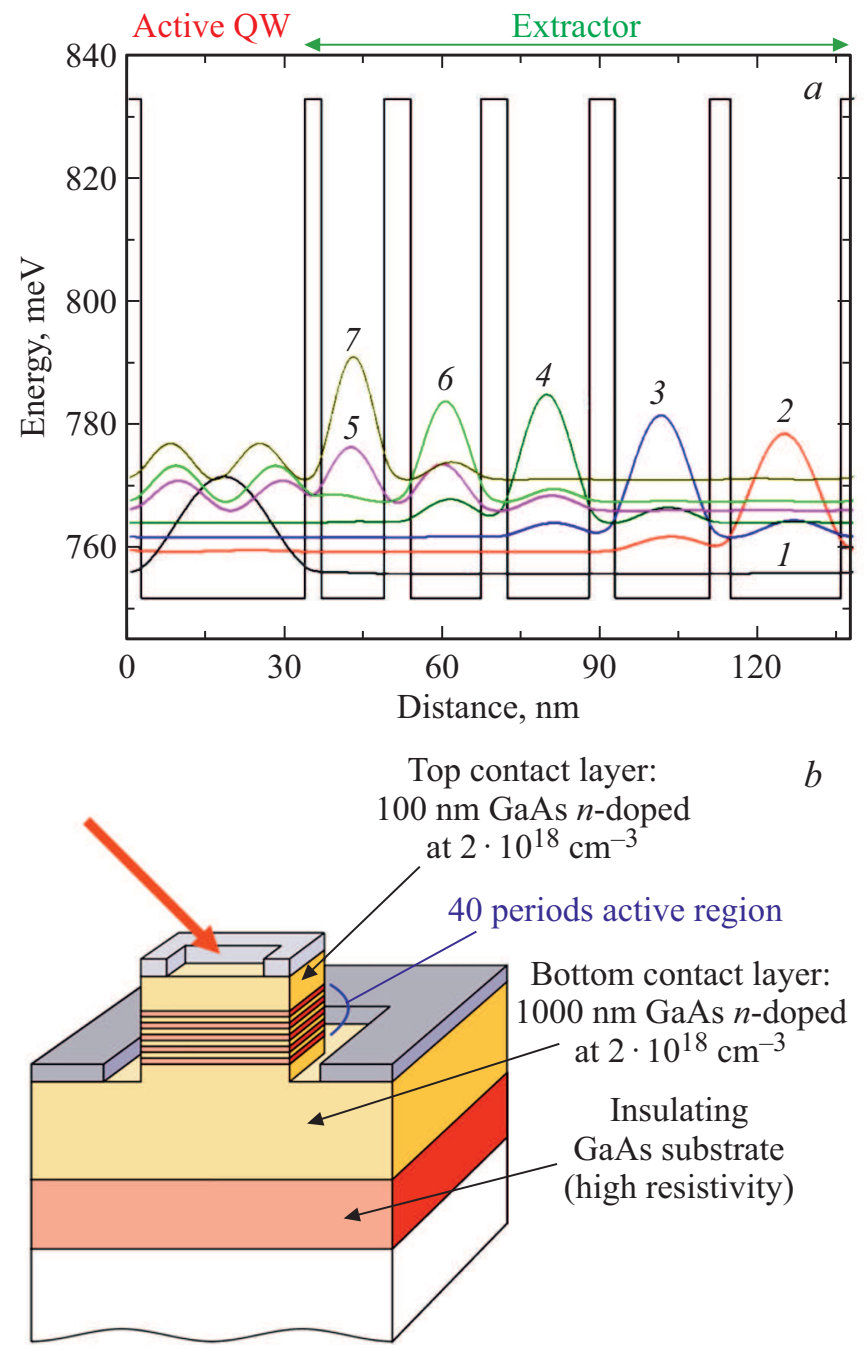

Рис. 1. $a$ - результаты численного расчета квадрата волновых функций в периоде каскада ККД частотного диапазона 2.5 ТГц; $b$ - схематическое изображение конструкции кристалла ККД. Стрелкой отмечено направление излучения, падающего под углом Брюстера. (Цветной вариант рисунка представлен в электронной версии статьи).

периодами каскада. Уровень легирования КЯ подбирался таким образом, чтобы, с одной стороны, увеличить вероятность межподзонного поглощения в ,активной“ КЯ, а с другой - исключить заполнение последнего состояния в области экстрактора. Детальное описание слоев гетероструктуры ККД представлено в табл. 1. Слои активной области и области экстрактора, выделенные жирным шрифтом, формируют один период каскада. Количество периодов каскада - 40. Суммарная толщина слоев в периоде каскада - $1378.5 \AA$. Подчеркнутые слои GaAs (в области экстрактора) легированы кремнием (объемное легирование).

Гетероструктура ККД была выращена методом молекулярно-пучковой эпитаксии (МПЭ). В качестве подложки использовались пластины $\mathrm{GaAs}$ с ориентацией $(001) \pm 0.5^{\circ}$, тип проводимости - полуизолятор (для 
Таблица 1. Описание слоев гетероструктуры ККД

\begin{tabular}{c|c|c}
\hline Материал & $\begin{array}{c}\text { Уровень } \\
\text { легирования, см }\end{array}$ & Толщина, $\AA$ \\
\hline $\mathrm{GaAs}$ & $2 \cdot 10^{18}$ & 1000 \\
$\mathrm{Al}_{0.10} \mathrm{GaAs}$ & $1 \cdot 10^{16}$ & 46 \\
$\underline{\mathrm{GaAs}}$ & $1 \cdot 10^{16}$ & 210 \\
$\mathrm{Al}_{0.10 \mathrm{GaAs}}$ & $1 \cdot 10^{16}$ & 40 \\
$\underline{\mathrm{GaAs}}$ & $1 \cdot 10^{16}$ & 50 \\
$\mathrm{Al}_{0.10 \mathrm{GaAs}}$ & & 155 \\
$\mathrm{GaAs}$ & 50 \\
$\mathrm{Al}_{0.10} \mathrm{GaAs}$ & & 50 \\
$\mathrm{GaAs}$ & & 130 \\
$\mathrm{Al}_{0.10} \mathrm{GaAs}$ & & 30 \\
$\mathrm{GaAs}$ & $2 \cdot 10^{18}$ & 412.5 \\
$\mathrm{Al}_{0.10} \mathrm{GaAs}$ & & 10000 \\
$\mathrm{GaAs}$ & & $350 \mathrm{Mкм}$ \\
$\mathrm{Al}_{0.10 \mathrm{GaAs}}$ & &
\end{tabular}

уменьшения потерь на свободных носителях заряда). Слой $\mathrm{GaAs}$ толщиной 1 мкм, легированный кремнием до уровня $n=2 \cdot 10^{18} \mathrm{~cm}^{-3}$, выполнял функцию нижнего контактного слоя. Активная область включала 40 периодов каскада на основе гетеропары твердых растворов $\mathrm{GaAs} / \mathrm{Al}_{0.1} \mathrm{Ga}_{0.9} \mathrm{As}$. Суммарная толщина слоев в одном периоде каскада составила 137.9 нм. Верхний контактный слой сформирован на основе GaAs c уровнем легирования $2 \cdot 10^{18} \mathrm{~cm}^{-3}$. Схематическое изображение конструкции кристалла ККД представлено на рис. $1, b$. Выбрана конструкция, в которой нижний контакт осуществляется к сильно легированному слою $\mathrm{GaAs}$, a не к легированной подложке, с целью уменьшения оптических потерь, связанных с использованием сильно легированной подложки. Верхний контакт предполагается формировать в виде рамки по периметру мезы для засветки образца под углом Брюстера.

Для оценки структурного качества гетероструктуры ККД использовались методы рентгенодифракционного анализа и просвечивающей электронной микроскопии (ПЭМ). ПЭМ-исследования проводились на просвечивающем электронном микроскопе JEM2100F (Jeol, Япония) с ускоряющим напряжением 200 кВ. Использовалась стандартная методика подготовки образцов в геометрии поперечного сечения, которая представлена, например, в работах $[18,19]$.

Измерения рентгенодифракционных кривых качания были проведены вблизи симметричного рефлекса (004) GaAs на дифрактометре PANalytical X'PertPro в параллельной геометрии пучка рентгеновского излучения [18]. Источником рентгеновского излучения мощностью 6 кВт является трубка с вращающимся медным анодом $(\lambda=0.15406$ нм). Полуширина первичного пучка не превышала $12^{\prime \prime}$, что обеспечивалось использованием четырехкратного Ge (220) прорезного кристалла монохроматора.

\section{3. Результаты и обсуждение}

Предварительные калибровки скоростей роста GaAs и AlAs, а также состава слоя $\mathrm{Al}_{0.1} \mathrm{Ga}_{0.9} \mathrm{As}$ были проведены на основе данных, полученных методом рентгеновской дифракции на тестовой гетероструктуре с объемным слоем AlGaAs. В результате были определены скорости роста GaAs и AlAs как 2.08 и $0.23 \AA$ /с соответственно. Для точной калибровки толщин тонких слоев в периодах каскада ККД непосредственно перед эпитаксиальным ростом гетероструктуры ККД проведен рост тестовой гетероструктуры, идентичной гетероструктуре ККД, но с уменьшенным до 10 числом периодов каскада. Произведено измерение рентгенодифракционной кривой качания $\Omega-2 \theta$ тестовой гетероструктуры. На кривой наблюдается наличие пиков-сателлитов высших порядков, характерных для периодической структуры периодов каскада. Анализ положения пиков-сателлитов показал, что средняя толщина периода каскада составила $139.0 \pm 6$ нм, что свидетельствует об отклонении от ростовой спецификации. По этой причине для коррекции толщины периода каскада при эпитаксиальном росте гетероструктуры ККД в ростовой программе скорость по галлию в слоях сверхрешетки была увеличена с 2.08 до $2.11 \AA$ /с. Уровень легирования был откалиброван методом вольт-емкостного профилирования тестовых гетероструктур с толстыми слоями GaAs: $\mathrm{Si}$.

Рост гетероструктуры ККД проводился при температуре подложки $575^{\circ} \mathrm{C}$ и соотношении потоков материалов III группы и As 1:10. Температура подложки контролировалась по показаниям пирометра, откалиброванного на температуре начала сгона окисла с подложки $\mathrm{GaAs}$, принятой за $590^{\circ} \mathrm{C}$. Скорость вращения подложкодержателя составляла 20 оборотов в минуту, что при выбранных скоростях роста обеспечивало более

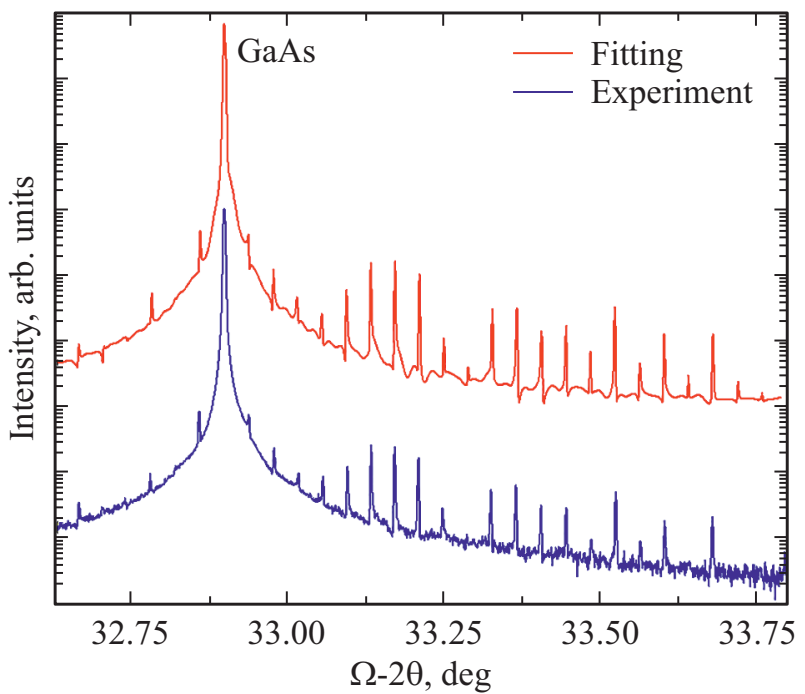

Рис. 2. Рентгенодифракционная кривая качания гетероструктуры ККД, измеренная в центре пластины (нижняя кривая), наряду с результатами численного моделирования (верхняя кривая). 

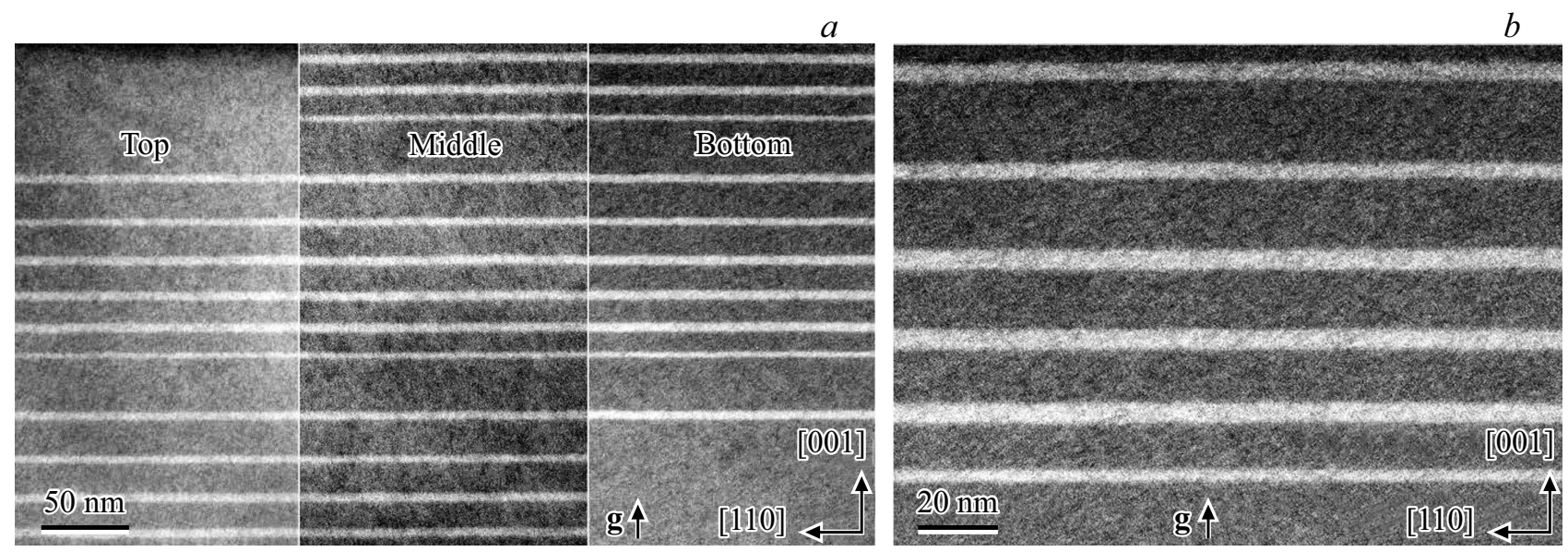

Рис. 3. $а$ - темнопольные изображения поперечного сечения (110) образца в двулучевых условиях с действующим вектором дифракции $g=(002)$ вблизи поверхности гетероструктуры (обозначение „тор“), в центральной части активной области (обозначение „middle“) и вблизи подложки (обозначение „,bottom“); $b$ - темнопольное изображение поперечного сечения образца с действующим вектором дифракции $g=(002)$ в центральной части активной области.

5 оборотов за время роста самого тонкого слоя периода каскада. Тем самым обеспечивалась лучшая однородность толщин слоев по площади подложки.

На рис. 2 представлена рентгенодифракционная кривая качания $\Omega-2 \theta$ гетероструктуры ККД, измеренная в центре пластины (нижняя кривая) наряду с результатами численного моделирования. На кривой качания наблюдается полное совпадение нулевого пика сателлитной структуры с положением пика подложки GaAs (обозначение „GaAs“). Данный факт свидетельствует о точном соответствии химического состава эпитаксиальных слоев заданным в ростовой спецификации. На кривой качания наблюдается наличие пиков-сателлитов высших порядков (вплоть до 21 порядка). Анализ кривой дает достаточно малое значение средней ширины на половине высоты (FWHM) пиков-сателлитов $(8.3 \pm 0.5)^{\prime \prime}$ по сравнению с ранее представленными результатами для подобных гетероструктур ТГЦ ККЛ на подложках GaAs $\left(13-19^{\prime \prime}\right)$ [20-23], что свидетельствует о высокой резкости границ, постоянстве толщин периодов каскада и слоев в них, а также о малой шероховатости гетероинтерфейсов в исследуемой гетероструктуре [23]. На основании положения 10 пиков-сателлитов была проведена оценка средней толщины периода каскада, которая составила $135.0 \pm 2.0$ нм, что соотносится с суммарной толщиной слоев периода каскада, заданной в ростовой спецификации (137.85 нм). Численная аппроксимация положения всех пиков-сателлитов кривой качания дает значение средней толщины периода каскада 135.1 нм. Проведено измерение рентгенодифракционных кривых на удалении в 25 мм от центра подложки. Согласно результатам моделирования, отклонение средней толщины периода каскада не превышает $1 \%$ в сравнении с центром подложки, что свидетельствует о высокой однородности слоев по поверхности гетероструктуры. Таким образом, данные рентгенодифракционного анали- за свидетельствуют о высоком структурном совершенстве созданной гетероструктуры ККД и соответствии толщин и составов слоев в периодах каскада заданным в ростовой спецификации.

Анализ результатов исследования темнопольного ПЭМ-изображения поперечного сечения (1피) образца c $g=(002)$ вблизи подложки методом просвечивающей электронной микроскопии показал отсутствие границы между подложкой и эпитаксиальными слоями. На рис. 3, а приведено ПЭМ-изображение периодов каскада для трех характерных областей гетероструктуры ККД: вблизи поверхности гетероструктуры, в центре активной области и вблизи подложки. В пределах периода различий в толщине слоев нет и все слои периода каскада имеют планарные гетерограницы, что представлено на увеличенном изображении (см. рис. $3, b$ ). Толщина однотипных слоев в периодах каскада не меняется в

Таблица 2. Результаты определения толщин слоев в периоде каскада методом ПЭМ. Приборная погрешность, согласно методике измерений, составляет $\pm 5 \%$

\begin{tabular}{c|c|c}
\hline Материал & $\begin{array}{c}\text { Толщина } \\
\text { по дизайну, } \AA\end{array}$ & $\begin{array}{c}\text { Измеренная ПЭМ } \\
\text { толщина, } \AA\end{array}$ \\
\hline $\mathrm{GaAs}$ & 210 & 210 \\
$\mathrm{Al}_{0.1} \mathrm{Ga}_{0.9} \mathrm{As}$ & 40 & 42 \\
$\mathrm{GaAs}$ & 180 & 180 \\
$\mathrm{Al}_{0.1} \mathrm{Ga}_{0.9} \mathrm{As}$ & 50 & 50.2 \\
$\mathrm{GaAs}$ & 155 & 154 \\
$\mathrm{Al}_{0.1} \mathrm{Ga}_{0.9} \mathrm{As}$ & 50 & 50.2 \\
$\mathrm{GaAs}$ & 135 & 133 \\
$\mathrm{Al}_{0.1} \mathrm{Ga}_{0.9} \mathrm{As}$ & 50 & 50.2 \\
$\mathrm{GaAs}$ & 120 & 117 \\
$\mathrm{Al}_{0.1} \mathrm{Ga}_{0.9} \mathrm{As}$ & 30 & 29.7 \\
$\mathrm{GaAs}$ & 312.5 & 312 \\
$\mathrm{Al}_{0.1} \mathrm{Ga}_{0.9} \mathrm{As}$ & 46 & 45.1
\end{tabular}


процессе роста, протяженных дефектов не обнаружено. Толщины слоев в периоде каскада, полученные на основе анализа ПЭМ-изображений, представлены в табл. 2. Суммарная толщина слоев в периоде каскада составила $(137.3 \pm 6.9)$ нм.

\section{4. Заключение}

В результате численного моделирования гетероструктуры квантово-каскадного детектора частотного диапазона 2.5 ТГц методом итерационного решения уравнения Шредингера-Пуассона в $k \cdot p$ формализме определены оптимальные толщины слоев активной области гетероструктуры (конструкция гетероструктуры квантово-каскадного детектора). Проведена отработка эпитаксиальных условий выращивания методом молекулярно- пучковой эпитаксии гетероструктур ККД в соответствии с предложенной конструкцией ККД. Результаты рентгеноструктурного анализа и просвечивающей электронной микроскопии подтверждают высокое структурное совершенство изготовленной гетероструктуры и соответствие параметрам расчетной конструкции ККД.

\section{Финансирование работы}

Работа выполнена при финансовой поддержке гранта Российского научного фонда (проект № 20-79-10285).

\section{Конфликт интересов}

Авторы заявляют, что у них нет конфликта интересов.

\section{Список литературы}

[1] L. Consolino, S. Bartalini, H. Beere, D. Ritchie, M. Vitiello, P. De Natale. Sensors, 13 (3), 3331 (2013).

[2] G.-R. Kim, T.-I. Jeon, D. Grischkowsky. Opt. Express, 25 (21), 25422 (2017).

[3] M. Locatelli, M. Ravaro, S. Bartalini, L. Consolino, M.S. Vitiello, R. Cicchi, F. Pavone, P. De Natale. Sci. Rep., 5 (1), 13566 (2015).

[4] N. Rothbart, O. Holz, R. Koczulla, K. Schmalz, H.-W. Hübers. Sensors, 19 (12), 2719 (2019).

[5] P.U. Jepsen, D.G. Cooke, M. Koch. Laser Photon. Rev., 5 (1), 124 (2010).

[6] A. Khalatpour, A.K. Paulsen, C. Deimert, Z.R. Wasilewski, Q. Hu. Nature Photonics, 15 (1), 16 (2020).

[7] A. Vardi, N. Kheirodin, L. Nevou, H. Machhadani, L. Vivien, P. Crozat, M. Tchernycheva, R. Colombelli, F.H. Julien, F. Guillot, C. Bougerol, E. Monroy, S. Schacham, G. Bahir. Appl. Phys. Lett., 93 (19), 193509 (2008).

[8] M. Hakl, Q. Lin, S. Lepillet, M. Billet, J.-F. Lampin, S. Pirotta, R. Colombelli, W. Wan, J.C. Cao, H. Li, E. Peytavit, S. Barbieri. ACS Photonics, 8 (2), 464 (2021).
[9] D. Palaferri, Y. Todorov, Y.N. Chen, J. Madeo, A. Vasanelli, L.H. Li, A.G. Davies, E.H. Linfield, C. Sirtori. Appl. Phys. Lett., 106 (16), 161102 (2015).

[10] H. Li, W.-J. Wan, Z.-Y. Tan, Z.-L. Fu, H.-X. Wang, T. Zhou, Z.-P. Li, C. Wang, X.-G. Guo, J.-C. Cao. Sci. Rep., 7 (1), 3452 (2017).

[11] B. Paulillo, S. Pirotta, H. Nong, P. Crozat, S. Guilet, G. Xu, S. Dhillon, L.H. Li, A.G. Davies, E.H. Linfield, R. Colombelli. Optica, 4 (12), 1451 (2017).

[12] P. Micheletti, J. Faist, T. Olariu, U. Senica, M. Beck, G. Scalari. APL Phot. Optica, 6, 106102 (2021).

[13] M. Graf, G. Scalari, D. Hofstetter, J. Faist, H. Beere, E. Linfield, D. Ritchie, G. Davies. Appl. Phys. Lett., 84 (4), 475 (2004).

[14] J. Popp, M. Haider, M. Franckie, J. Faist, C. Jirauschek. In: 2020 Int. Conf. on Numerical Simulation of Optoelectronic Devices (NUSOD) (Turin, Italy, 2020). doi: 10.1109/NUSOD49422.2020.9217784

[15] J. Popp, M. Haider, M. Franckie, J. Faist, C. Jirauschek. In: 2020 XXXIIIrd General Assembly and Scientific Symposium of the Int. Union of Radio Science (Rome, Italy, 2020). doi: 10.23919/URSIGASS49373.2020.9232167

[16] J. Popp, M. Haider, M. Franckié, J. Faist, C. Jirauschek. Opt. Quant. Electron., 53 (6), 287 (2021).

[17] link: https://www.nextnano.de/. Accepted date: 21.09.2021.

[18] A.V. Babichev, V.V. Dudelev, A.G. Gladyshev, D.A. Mikhailov, A.S. Kurochkin, E.S. Kolodeznyi, V.E. Bougrov, V.N. Nevedomskiy, L.Y. Karachinsky, I.I. Novikov, D.V. Denisov, A.S. Ionov, S.O. Slipchenko, A.V. Lutetskiy, N.A. Pikhtin, G.S. Sokolovskii, A.Y. Egorov. Techn. Phys. Lett., 45 (7), 735 (2019).

[19] A.V. Babichev, A.S. Kurochkin, E.C. Kolodeznyi, A.V. Filimonov, A.A. Usikova, V.N. Nevedomsky, A.G. Gladyshev, L.Y. Karachinsky, I.I. Novikov, A.Y. Egorov. Semiconductors, 52 (6), 745 (2018).

[20] A.E. Zhukov, G.E. Cirlin, R.R. Reznik, Y.B. Samsonenko, A.I. Khrebtov, M.A. Kaliteevski, K.A. Ivanov, N.V. Kryzhanovskaya, M.V. Maximov, Z.I. Alferov. Semiconductors, 50 (5), 662 (2016).

[21] G.E. Cirlin, R.R. Reznik, A.E. Zhukov, R.A. Khabibullin, K.V. Maremyanin, V.I. Gavrilenko, S.V. Morozov. Semiconductors, 54 (9), 1092 (2020).

[22] H.E. Beere, J.R. Freeman, O.P. Marshall, C.H. Worrall, D. Ritchie. J. Cryst. Growth, 311 (7), 1923 (2009).

[23] X. Wang, C. Shen, T. Jiang, Z. Zhan, Q. Deng, W. Li, W. Wu, N. Yang, W. Chu, S. Duan. AIP Adv., 6 (7), 075210 (2016).

Редактор Г.А. Оганесян 


\title{
Heterostructure of a $2.5 \mathrm{THz}$ range quantum-cascade detector
}

A.V. Babichev ${ }^{1}$, E.S. Kolodeznyi ${ }^{1}$, A.G. Gladyshev ${ }^{1}$, D.V. Denisov ${ }^{2}$, A. Jollivet ${ }^{3}$, P. Quach ${ }^{3}$,

L.Ya. Karachinsky $1,4,5$, V.N. Nevedomskiy ${ }^{4}$, I.I. Novikov ${ }^{1,5}$, M. Tchernycheva ${ }^{3}$, F.H. Julien ${ }^{3}$, A.Yu. Egorov ${ }^{5}$

1 ITMO University, 197101 St. Petersburg, Russia

${ }^{2}$ St. Petersburg Electrotechnical University „LETl“, 197022 St. Petersburg, Russia

${ }^{3}$ Centre de Nanosciences

et de Nanotechnologies (C2N),

UMR 9001 CNRS, Universite Paris-Saclay,

91120 Palaiseau, France

${ }^{4}$ loffe Institute,

194021 St. Petersburg, Russia

${ }^{5}$ Connector Optics LLC, 194292 St. Petersburg, Russia

\begin{abstract}
The design of the heterostructure of a $2.5 \mathrm{THz}$ range quantum-cascade detector is proposed and heterostructure is grown by molecular-beam epitaxy technique. To optimize the thicknesses of the layers of the heterostructure cascades, a numerical method for iterative solution of the Schrödinger-Poisson equation in the $k \cdot p$ formalism was used. The grown heterostructure of the quantum-cascade detector showed a high structural perfection, confirmed by the small values of the average FWHM of the high-order satellite peaks on the $X$-ray diffraction rocking curves, which were $(8.3 \pm 0.5)^{\prime \prime}$. Analysis of dark-field images obtained by transmission electron microscopy showed that the total thickness of the layers in the cascade is $(137.3 \pm 6.9) \mathrm{nm}$, which corresponds to the calculated thickness of the layers in the cascade of the heterostructure of the quantum-cascade detector.
\end{abstract}

